\title{
Comparison of regression models in case of non-normality and Heteroscedasticity
}

\author{
Seray Kahvecioglu, Berna Yazici* \\ Anadolu University, Science Faculty, Department of Statistics, Eskisehir, Turkey
}

\section{A R T I C LE I N F O}

\section{Article history:}

Received 4 July 2016

Received in revised form

14 August 2016

Accepted 14 August 2016

\section{Keywords:}

Generalized $\mathrm{p}$ values

Generalized $p$ values in regression

Regression analysis in case of

Assumption violation

Comparison of regression coefficients

\begin{abstract}
A B S T R A C T
In regression analysis, in case of comparing two regression models and coefficients where the distribution of variables in question is not known, generalized $p$ values may be used. The generalized $p$ value is an extended version of the classical $p$ value which provides only approximate solutions. Use of approximate methods, generalized $p$ value, has better results, performance with small samples. In this study, the generalized $p$ value which may be used alternatively when different assumptions aren't fulfilled is researched theoretically; a simulation is conducted and an application in regression analysis is given. It is concluded that in generalized p value works well for the comparison of regression coefficients both under non-normality and heteroscedasticity.
\end{abstract}

(C) 2016 The Authors. Published by IASE. This is an open access article under the CC BY-NC-ND license (http://creativecommons.org/licenses/by-nc-nd/4.0/).

\section{Introduction}

Comparing two or more regression lines is a common problem in statistical application studies. Assumption violation has been a popular topic which has been studied by numerous researchers. Generalized $p$ value theory is one of them that are commonly used particularly for non-normality and heteroscedasticity; (Tsui and Weerahandi, 1989; Weerahandi, 2013; Sezer et al., 2015) comparing the regression models is frequently in question when researcher aims to see the change in regression equation or in regression parameters. In this study, generalized $p$ value theory which is used in case of assumption violation is examined. Especially the homogeneity of regression models is examined under heteroscedasticity. A simulation study is conducted in case of assumption violation for comparison of regression models. Also an application is given for a small sample for assumption violation.

\section{Data and methods}

The concept of generalized test variable and generalized $\mathrm{p}$ value is firstly proposed by Tsui and Weerahandi (1989). In that commonly used method,

\footnotetext{
* Corresponding Author. Tel.: +90 5369222434

Email Addresses: s.mankir@hotmail.com (S. Kahvecioglu), bbaloglu@anadolu.edu.tr (B. Yazici)

http://dx.doi.org/10.21833/ijaas.2016.08.004

2313-626X/C 2016 The Authors. Published by IASE

This is an open access article under the CC BY-NC-ND license

(http://creativecommons.org/licenses/by-nc-nd/4.0/)
}

when the distribution of the variables' is unknown the $\mathrm{p}$-value is being calculated via iterative calculations.

\subsection{Comparison of two regression models with homogenous variances}

For two regression models, while some of the parameters are different, some of them maybe common. Hence let's, take into account the following linear regression model:

$y_{j}=U_{j} c+V_{j} d_{j}+W_{j} t_{j}+\varepsilon_{j}, \quad j=1,2$

Suppose that the normality assumption is valid;

$\varepsilon_{i} \sim N\left(0, \sigma_{j}^{2} I_{j}\right), \quad i=1,2, \ldots, n$

it is assumed that $\sigma_{1}^{2}=\sigma_{2}^{2}$ for $I_{j}, j=1,2$, . The lengths of vectors $y_{1}$ and $y_{2}$ are defined by the dependent variables with $n_{1}$ and $n_{2}$ observations. We will denote the explanatory variables with matrices $U_{j}, V_{j}$ and $W_{j}$. While the variables are common for the matrixes $U_{j}$ and $W_{j}$, the variables for $V_{1}$ and $V_{2}$ are different from each other. For the response vectors:

$y^{\prime}=\left(y_{1}^{\prime}, y_{2}^{\prime}\right)$

and the design matrices: 
$X_{12}=\left[\begin{array}{cclll}U_{1} & 0 & V_{1} & 0 & W_{1} \\ 0 & U_{2} & 0 & V_{2} & W_{2}\end{array}\right] \quad\left(n_{1}+n_{2}\right) \times$

$\left(2 p_{c}+p_{d_{1}}+p_{d_{2}}+p_{t}\right)$

and

$X_{1,2}=\left[\begin{array}{cccc}U_{1} & 0 V_{1} & 0 W_{1} & 0 \\ 0 & U_{2} 0 & V_{2} 0 & W_{2}\end{array}\right] \quad\left(n_{1}+n_{2}\right) \times$

$\left(2 p_{c}+p_{d_{1}}+p_{d_{2}}+2 p_{t}\right)$

1,2 are used for $t_{1} \neq t_{2}$ while, 12 is used for $t_{1}=t_{2}$. Where $\mathrm{c}$ is known parameter vector and the length of it is $p_{c}$. The lenghts of $d_{1}$ and $d_{2}$ are $p_{d_{1}}$ and $p_{d_{2}}$, respectively. These also include the parameters that are expected to be different. In other words, they are the coefficients of the variables that consist the matrixes of $V_{1}$ and $V_{2}$ which are coming from different distributions. In order to test the equality of $t_{1}$ and $t_{2}$ vectors, denoting the lengths of the vectors as $p_{t}$.

Under $H_{0}: t_{1}=t_{2}$, we test the equality of coefficients of two different regression models.

The sum of squares residual of the matrixes are $s_{12}^{2}, s_{1,2}^{2}$ respectively. If there is not common parameters of the regression model, sum of squares residual is calculated by summing the sums of squares residual separately; $s_{1,2}^{2}=s_{1}^{2}+s_{2}^{2}$.

$F=\frac{\left(s_{12}^{2}-s_{1,2}^{2}\right) / p_{t}}{s_{1,2}^{2} / l} \sim F_{p_{t}, l}$

$l=\left(n_{1}+n_{2}\right)-\left(2 p_{c}+p_{d_{1}}+p_{d_{2}}+2 p_{t}\right)$

$p=1-H_{p_{t}, l}\left[\frac{\left(s_{12}^{2}-s_{1,2}^{2}\right) / p_{t}}{s_{1,2}^{2} / l}\right]$

$H_{k, l}$ is the cumulative distribution function of $\mathrm{F}$ distribution with degrees of freedom $k$ and $l$. If $p<$ $\alpha$, then $H_{0}$ is rejected.

\subsection{Comparison of two regression models without common parameters}

While the comparison of two regression models, if the unknown parameter situation is in question, in other words, let us c $=0$ in the model. In order to get the unbiased tests depending on the generalized $\mathrm{p}$ value, $\tilde{y}_{j}=a_{j}^{-1} y_{j}, \tilde{V}_{j}=a_{j}^{-1} V_{j}$ and $\widetilde{W}_{j}=a_{j}^{-1} W_{j}$. $a_{1}^{2}=s_{1}^{2} / R$ and $a_{2}^{2}=s_{2}^{2} /(1-R)$ (where $\mathrm{R}$ is Beta distributed parameter.)

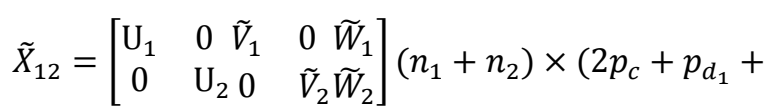

$\left.p_{d_{2}}+p_{t}\right)$,

and

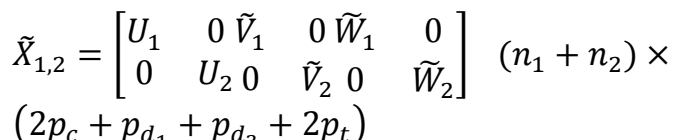

In order to test the hypothesis, the $\mathrm{p}$ value is obtained as follows (Weerahandi, 2013):

$p=1-E_{R}\left\{H_{k, l}\left[\frac{l}{k}\left(\tilde{s}_{12}^{2}\left(\frac{s_{1}^{2}}{R}, \frac{s_{2}^{2}}{(1-R)}\right)-1\right)\right]\right\}$

$\tilde{s}_{12}^{2}, a_{1}^{2}=s_{1}^{2} / R$ ve $a_{2}^{2}=s_{2}^{2} /(1-R) k=p_{t}, l=$

$n_{1}+n_{1}-\left(2 p_{c}+p_{d_{1}}+p_{d_{2}}+2 p_{t}\right)$

where $H_{k . l}$ is the cumulative distribution function of $F$ distribution with degrees of freedoms $k$ and $l$, and the expected values are obtained from Beta distribution as follows (Weerahandi, 2013):

$R \sim \operatorname{Beta}\left(\frac{n_{1}-2 p_{t}-p_{d_{1}}}{2}, \frac{n_{2}-2 p_{t}-p_{d_{2}}}{2}\right)$

Beta distributed $\mathrm{R}$ will be used to obtain the $\mathrm{p}$ value in order to compare the regression coefficients.

\subsection{Comparison of two regression models with heterogeneous variances}

Two regression models are defined as follows. Let us assume their variances are not equal $\left(\sigma_{1}^{2} \neq \sigma_{2}^{2}\right)$ and the sample size is moderate.

$y_{j}=U_{j} c+V_{j} d_{j}+W_{j} t_{j}+\varepsilon_{j}, \quad j=1,2$

$\tilde{X}_{12}=\left[\begin{array}{ccccc}\widetilde{U}_{1} & 0 & \widetilde{V}_{1} & 0 & \widetilde{W}_{1} \\ 0 & \widetilde{U}_{2} 0 & \tilde{V}_{2} \widetilde{W}_{2}\end{array}\right], \quad\left(n_{1}+n_{2}\right) \times$

$\left(2 p_{c}+p_{d_{1}}+p_{d_{2}}+p_{t}\right)$

and

$\tilde{X}_{1,2}=\left[\begin{array}{cccccc}\widetilde{U}_{1} & 0 & \tilde{V}_{1} & 0 & \widetilde{W}_{1} & 0 \\ 0 & \widetilde{U}_{2} & 0 & \tilde{V}_{2} & 0 & \widetilde{W}_{2}\end{array}\right], \quad\left(n_{1}+n_{2}\right) \times$

$\left(2 p_{c}+p_{d_{1}}+p_{d_{2}}+2 p_{t}\right)$

In order to compare the regression coefficients of different regression models $\left(H_{0}: t_{1}=t_{2}\right)$, the test variable given below is calculated:

$T=\frac{\left(\tilde{S}_{12}^{2}-\tilde{S}_{1,2}^{2}\right)\left(\sigma_{1}^{2}, \sigma_{2}^{2}\right)}{\left(\tilde{s}_{12}^{2}-\tilde{S}_{1,2}^{2}\right)\left(s_{1}^{2} \sigma_{1}^{2} / S_{1}^{2}, s_{2}^{2} \sigma_{2}^{2} / S_{2}^{2}\right)}$

The p-value can be obtained using Beta distributed variable:

$p=1-E_{R_{1}, R_{2}}\left\{H_{k, l}\left[\frac{l}{k} R_{2} \tilde{s}^{2}\left(\frac{s_{1}^{2}}{R_{1}}, \frac{s_{2}^{2}}{\left(1-R_{1}\right)}\right)\right]\right\}$

$R_{1} \sim \operatorname{Beta}\left(\frac{n_{1}-p_{c}-p_{t}-p_{d_{1}}}{2}, \frac{n_{2}-p_{c}-p_{t}-p_{d_{2}}}{2}\right)$,

$R_{2} \sim \operatorname{Beta}\left(\frac{n_{1}+n_{2}-2 p_{c}-2 p_{t}-p_{d_{1}}-p_{d_{2}}}{2}, \frac{p_{c}}{2}\right)$.

$y_{j}=U_{j} c+V_{j} d_{j}+W_{j} t_{j}+\varepsilon_{j}, \quad j=1,2$

Generalized $p$ value is calculated in the comparison of regression coefficients, if the distributions of the variables which are used in the 
regression models are not known or when the homoscedasticity assumption is not valid. For the simulation studies, $\mathrm{R}$ is used. In order to conduct simulations, 8-unit samples are chosen from certain distributions. Those samples are used to obtain generalized $\mathrm{p}$ values with 500 replications using the design matrix. The obtained $p$ values are given in the following tables. $\mathrm{R}$ is used to conduct the simulation study.

Let us assume two regression models as given below and we are interested in comparing them. While $V_{1}$ in the first model is Normal $(0,2.5)$, and $W_{1}$ is Gamma $(2,2)$ distributed, $V_{2}$ in the second model is Weibul $(1,1.5)$, and $W_{2}$ is Gamma $(2,2)$ distributed.

$y_{j}=U_{j} c+V_{j} d_{j}+W_{j} t_{j}+\varepsilon_{j}, \quad j=1,2$

We suppose that those two models do not have the common parameters. The generalized $p$ values after 500 replications are given in Table 1.

When Table 1 is examined, it can be seen that, only 2 values are smaller or closed to nominal value 0.05. The null hypothesis can be accepted for remaining 98 values. In other words, according to Table 1, there is no significant difference between the two regression models.

The simulation scenario is changed for the comparison of two regression models. As can be seen from Table 2 that, in the first model $V_{1}$ is Normal $(0$, 2.5) distributed and $W_{1}$ is Gamma (9, 0.5) distributed. On the other hand in the second model, similarly $V_{2}$ is Normal $(0,2.5)$ and $W_{2}$ is Gamma $(9$, 0.5) distributed.

When the simulation results are examined for the second scenario in Table 2 , it can be seen that only 2 values are smaller than 0.05 or closed to it. As the result of the others, null hypothesis $H_{0}$ should be accepted. In other words, the difference the regression models constructed for Table 2 are not statistically significant.

In order to construct the table to see the power of the test, the first models' variables $V_{1}$ and $W_{1}$ are chosen from Gamma(2,2) and the second models' variables $V_{2}$ and $W_{2}$ are chosen from $\operatorname{Beta}(2,5)$ distributed populations. The two regression models, with 8 units and without any common parameter, are compared with 10,000 replications using $\mathrm{R}$. The results are summarized in Table 3.

It can be seen from Table 3 that almost all values are closed to 1 , which means that, the test to compare the regression models from different distributions is powerful.

\section{Application}

A dietitian is applying a program for 3 months. After the program, the weight loss (kg.) of the subjects' are noted and modeled also taking into account their ages (year), heights (cm.) and gender. 16 subjects, 8 males and 8 females, are randomly selected after the weight loss diet. The data set is shown in Table 4.

Model form for two genders:

$$
Y=\alpha+\beta H e i g t h+\gamma \text { Age }+\varepsilon
$$

The least squares estimates of the parameters of the two regressions estimated using the above data are respectively:

$$
\hat{\alpha}_{M}=-10.441, \hat{\beta}_{M}=0.110, \hat{\gamma}_{M}=0.031
$$

and

$$
\hat{\alpha}_{F}=-39.969, \hat{\beta}_{F}=0.293, \hat{\gamma}_{F}=-0.037
$$

Table 1: Number of observations 8, Variable $V_{1} \sim N(0,2.5), V_{2} \sim$ Weibull $(1,1.5), W_{1}$ and $W_{2} \sim$ Gamma $(2,2)($ Generalized $p$ values for 500 replicates)

\begin{tabular}{|l|l|l|l|l|}
\hline \multicolumn{5}{|c|}{ Generalized p values for 500 replicates } \\
\hline 0.8563 & 0.9484 & 0.8493 & 0.9247 & 0.9790 \\
\hline 0.5150 & 0.3005 & 0.6902 & 0.1937 & 0.4306 \\
\hline 0.5561 & 0.9829 & 0.7998 & 0.7484 & 0.3430 \\
\hline 0.7751 & 0.4706 & 0.9430 & 0.3357 & 0.9444 \\
\hline 0.6093 & 0.5218 & 0.1002 & 0.1016 & 0.2897 \\
\hline 0.0837 & 0.7206 & 0.3605 & 0.4529 & 0.4019 \\
\hline 0.7548 & 0.4253 & 0.5839 & 0.6500 & 0.7650 \\
\hline 0.9367 & 0.5483 & 0.7634 & 0.3068 & 0.1265 \\
\hline 0.6241 & 0.5555 & 0.3008 & 0.4745 & 0.6148 \\
\hline 0.0863 & 0.9463 & 0.3758 & 0.1375 & 0.9144 \\
\hline 0.6310 & 0.8814 & 0.2994 & 0.4900 & 0.9682 \\
\hline $\mathbf{0 . 0 3 6 8}$ & $\mathbf{0 . 0 1 8 6}$ & 0.8868 & 0.7659 & 0.1246 \\
\hline 0.1015 & 0.6196 & 0.6317 & 0.2656 & 0.5202 \\
\hline 0.7911 & 0.4672 & 0.3707 & 0.6429 & 0.3557 \\
\hline 0.5453 & 0.6247 & 0.6601 & 0.9529 & 0.8344 \\
\hline 0.8907 & 0.3795 & 0.6434 & 0.7684 & 0.8592 \\
\hline 0.4738 & 0.7820 & 0.6650 & 0.8782 & 0.0842 \\
\hline 0.2432 & 0.4913 & 0.8121 & 0.9177 & 0.7930 \\
\hline 0.9265 & 0.2534 & 0.4391 & 0.3391 & 0.7036 \\
\hline
\end{tabular}


Table 2: $\mathrm{V}_{1} \sim \operatorname{Normal}(0,2.5), \mathrm{W}_{1} \sim \operatorname{Gamma}(9,0.5) \mathrm{V}_{2} \sim \operatorname{Normal}(0,2.5), \mathrm{W}_{2} \sim \operatorname{Gamma}(9,0.5)$ (Generalized $\mathrm{p}$ values for 500 replicates)

\begin{tabular}{|c|c|c|c|c|}
\hline \multicolumn{5}{|c|}{ Generalized p values for 500 replicates } \\
\hline 0.5855 & 0.8378 & 0.1099 & 0.5307 & 0.9569 \\
\hline 0.9666 & 0.1453 & 0.3514 & 0.4958 & 0.5149 \\
\hline 0.5183 & 0.6842 & 0.4408 & 0.6349 & 0.7684 \\
\hline 0.3429 & 0.2207 & 0.4353 & 0.1939 & 0.5287 \\
\hline 0.7931 & 0.1277 & 0.3449 & 0.1648 & 0.5794 \\
\hline 0.2190 & 0.5311 & 0.0818 & 0.3802 & 0.6908 \\
\hline 0.6637 & 0.5502 & 0.6817 & 0.8831 & 0.6387 \\
\hline 0.9748 & 0.3979 & 0.5839 & 0.1657 & 0.1899 \\
\hline 0.4438 & 0.4581 & 0.9608 & 0.6348 & 0.2271 \\
\hline 0.6384 & 0.2654 & 0.2339 & 0.3753 & 0.6532 \\
\hline 0.5094 & 0.5385 & 0.5070 & 0.1233 & 0.5852 \\
\hline 0.7218 & 0.2046 & 0.5413 & 0.7129 & 0.1634 \\
\hline 0.2456 & 0.3525 & 0.7753 & 0.9282 & 0.4027 \\
\hline 0.3627 & 0.8557 & 0.6640 & $\mathbf{0 . 0 4 0 2}$ & $\mathbf{0 . 0 4 6 0}$ \\
\hline 0.8611 & 0.1410 & 0.5164 & 0.3807 & 0.6450 \\
\hline 0.2513 & 0.7480 & 0.9542 & 0.1612 & 0.9121 \\
\hline 0.3221 & 0.4979 & 0.1229 & 0.4538 & 0.7499 \\
\hline 0.4753 & 0.5583 & 0.2122 & 0.1015 & 0.7901 \\
\hline 0.9552 & 0.5857 & 0.2674 & 0.7621 & 0.8563 \\
\hline 0.3709 & 0.2110 & 0.1607 & 0.4079 & 0.9283 \\
\hline
\end{tabular}

Table 3: $\mathrm{V}_{1} \sim$ Gamma $(2,2), \mathrm{V}_{2} \sim \operatorname{Beta}(2,5), \mathrm{W}_{1} \sim \operatorname{Gamma}(2,2)$ and $\mathrm{W}_{2} \sim \operatorname{Beta}(2,5)$ (Power of the test for 10000 replicates)

\begin{tabular}{|l|l|c|c|c|}
\hline \multicolumn{5}{|c|}{ Power of the test for $\mathbf{1 0 0 0 0}$ replicates } \\
\hline 0.6500 & 0.7083 & 0.5832 & 0.8457 & 0.8565 \\
\hline 0.9693 & 0.8446 & 0.9105 & 0.8455 & 0.9759 \\
\hline 0.8386 & 0.9040 & 0.9690 & 0.9178 & 0.9780 \\
\hline 0.8052 & 0.8184 & 0.9995 & 0.8123 & 0.9943 \\
\hline
\end{tabular}

Table 4: Data set for weight loss application

\begin{tabular}{|c|c|c|c|c|c|c|c|c|}
\hline & \multicolumn{8}{|c|}{ MALE } \\
\hline Age & 33 & 47 & 21 & 65 & 27 & 57 & 45 & 25 \\
\hline Height & 180 & 176 & 196 & 180 & 179 & 176 & 177 & 165 \\
\hline \multirow{2}{*}{ Weigth Loss } & 11.3 & 15.6 & 11.8 & 11 & 8.9 & 8.8 & 8.7 & 8.1 \\
\hline & \multicolumn{8}{|c|}{ FEMALE } \\
\hline Age & 39 & 44 & 19 & 23 & 33 & 27 & 31 & 69 \\
\hline Height & 162 & 164 & 164 & 164 & 154 & 162 & 164 & 158 \\
\hline Weigth Loss & 6.5 & 7.4 & 8.1 & 8.7 & 4.2 & 4.9 & 5.4 & 6 \\
\hline
\end{tabular}

The residual sums of squares from these regressions are $s_{1}^{2}=36.308$ and $s_{2}^{2}=8.743$, respectively. since the two regressions have no common parameters,

$s_{1,2}^{2}=s_{1}^{2}+s_{2}^{2}=36.308+8.743=45.051$.

Under null hypothesis;

$H_{0}: \hat{\beta}_{M}=\hat{\beta}_{F}$,

the residual sum of squares $s_{12}^{2}$ is to be computed by concatenated regressions of $Y$ on $X_{12}$.

The estimated parameters of this regression are

$$
\begin{gathered}
\hat{\alpha}_{M}=-15.823, \hat{\alpha}_{F}=-15.163, \hat{\beta}=0.0343, \hat{\gamma}_{M}= \\
-0.0346, \hat{\gamma}_{F}=0.139 . \\
s_{12}^{2}=47.707
\end{gathered}
$$

The observed value of the F-statistic appropriate for testing the null hypothesis can be computed as

$$
F=\frac{2.656 / 1}{45.051 / 10}
$$

Its $p$-value is $p=1-H_{1,10}(0.589)=0.4604$ to be computed by $\mathrm{R}$ language program, and therefore the data in Table 4 do not provide sufficient evidence to suspect the validity of the null hypothesis. The difference in weight loss for male and female is not significantly different.

\section{Conclusion}

In this study three different cases; comparison of regression models with common parameters, comparison of regression models without common parameters and comparison of parameters in case of heteroscedasticity are examined. For all cases, generalized $\mathrm{p}$ value method has given good results even in case of heteroscedasticity. It can be concluded that generalized $\mathrm{p}$ value can be used in cases of assumption violations; for both nonnormality and heteroscedasticity in order to compare the regression models. 


\section{References}

Sezer A, Ozkip E and Yazici B (2015). Comparison of confidence intervals for the behrens-fisher problem. Communications in StatisticsSimulation and Computation. DOI: 10.1080/03610918.2015.1082587.

Tsui KW and Weerahandi S (1989). Generalized pvalues in significance testing of hypotheses in the presence of nuisance parameters. Journal of the American Statistical Association, 84(406): 602607.

Weerahandi S (2013). Exact statistical methods for data analysis. Springer Science \& Business Media, New York, USA. 